\title{
Análise quantitativa de tensões em amostras fotoelásticas por meio de fotoelasticidade
}

Sidney Leal da Silva atec.professor.sidneyi@gmail.com Fatec Itaquera, São Paulo, SP, Brasil.

Mateus Aquino de Jesus mthsaqn@gmail.com,

Fatec Itaquera, São Paulo, SP, Brasil.

Charles Rodrigues da Silva

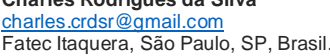

Daniel José Toffoli

Daniel José Tolfoli

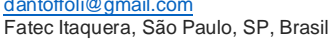

Uinguiston Nunes Camargo

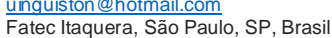

\section{RESUMO}

A Fotoelasticidade é uma ferramenta eficiente para determinação de tensões no interior de materiais fotoelásticos. Esses materiais apresentam a propriedade da dupla refração ou birrefringência temporária quando submetidos a esforços externos, que ocorre devido às alterações nos estados de polarizações da luz transmitida através de sua estrutura. A partir de um polariscópio linear de transmissão é possível obter imagens coloridas de franjas que são utilizadas para encontrar as diferenças de tensões por meio de análise com um método de Fotoelasticidade. 0 objetivo desse trabal ho foi determinar as distribuições dessas diferenças de tensões em duas franjas consecutivas de imagens monocromáticas produzidas em amostras fotoelásticas: pura e com um parafuso metálico inserido na estrutura, durante a cura. Os resultados apontam a possibilidade de uma análise quantitativa no estudo das propriedades por meio de um método da Fotoelasticidade, ligeiramente modificado em relação ao método tradicional.
\end{abstract}

PALAVRAS-CHAVE: M ateriais fotoelásticos; Fotoelasticidade; Polarização; Birrefringência. 


\section{INTRODUÇÃO}

A Fotoelasticidade é um ramo da óptica composto por técnicas e métodos que permitem investigar as tensões e deformações em materiais envolvidos com resinas que apresentam dupla refração ou birrefringência temporária. Estudar tensões e deformações e a forma com que elas agem em componentes diversos tornaram-se importante para fins científicos e tecnológicos. Por essa razão muitas técnicas e métodos foram e estão sendo produzidos com essa finalidade.

Nessa direção, a Fotoelasticidade apresenta técnicas e métodos amplamente explorados e de grande aceitação, como mostrados nos trabalhos de SWAIN et al. (2015), KALE e RAM ESH (2013) e ZHANG et al. (2012).

Com a computação, a dinâmica da análise torna-se rápida e os resultados podem ser obtidos de maneira sistemática e precisa. Os trabalhos de NETO et al. (2016), DA SILVA et al. (2015), M AIA et al. (2010) e CARLIN et al. (2005) mostram alguns exemplos de aplicações nas áreas de Engenharia de Mecânica, Física Aplicada, Odontologia e Biologia.

Nesse contexto, o propósito desse trabalho é apresentar um estudo sobre a distribuição de tensões internas em uma área selecionada de um material birrefringente temporário (resina fotoelástica), devido a esforços externos sobre metais inseridos nas resinas, a partir de franjas de interferência observadas em um polariscópio. Para a análise dos resultados será utilizado o tradicional método de fotoelasticidade RGB (Red, Green e Blue), ligeiramente modificado.

\section{BIRREFRINGÊNCIA}

A Birrefringência é o fenômeno associado à dupla refração intrínseca em materiais anisotrópicos como, por exemplo, a calcita $\left(\mathrm{CaCO}_{3}\right)$, ou temporária em materiais isotrópicos, como por exemplo a resina fotoelástica.

0 esquema geral da birrefringência, apresentado na Figura 1, mostra uma onda linearmente polarizada incide no material e se divide em onda-o, ordinária e onda-e, extraordinária, com índices de refração distintos, ao passar pelo plano que contém o eixo óptico do material devidamente crivado. As ondas ordinária e extraordinária emergentes possuem estados de polarizações ortogonais entre si.

Alguns cristais transparentes como a calcita $\left(\mathrm{CaCO}_{3}\right)$, possuem intrinsicamente a propriedade da dupla refração e, por isso, são denominados anisotrópicos. Esse fenômeno é denominado birrefringência. No esquema da Figura 1, a diferença de fase, $\Delta \phi$, entre as ondas ordinária e extraordinária está associada à diferença de caminho óptico, $\Delta r$, entre essas ondas, tal que:

$$
\Delta \phi=\mathrm{k} \cdot \Delta \mathrm{r} \Rightarrow \Delta \phi=\frac{2 \pi}{\lambda} \cdot \mathrm{e} \cdot\left|\mathrm{n}_{\mathrm{o}}-\mathrm{n}_{\mathrm{e}}\right|
$$

com $\mathrm{k}=2 \pi / \lambda$ representando número de onda; $\lambda$ o comprimento de onda da luz incidente; e é a espessura do material e $n_{0}$ e $n_{e} 0$ o índices de refração, ordinário e extraordinário, respectivamente. 
Figura 1 - Esquema geral da birrefringência

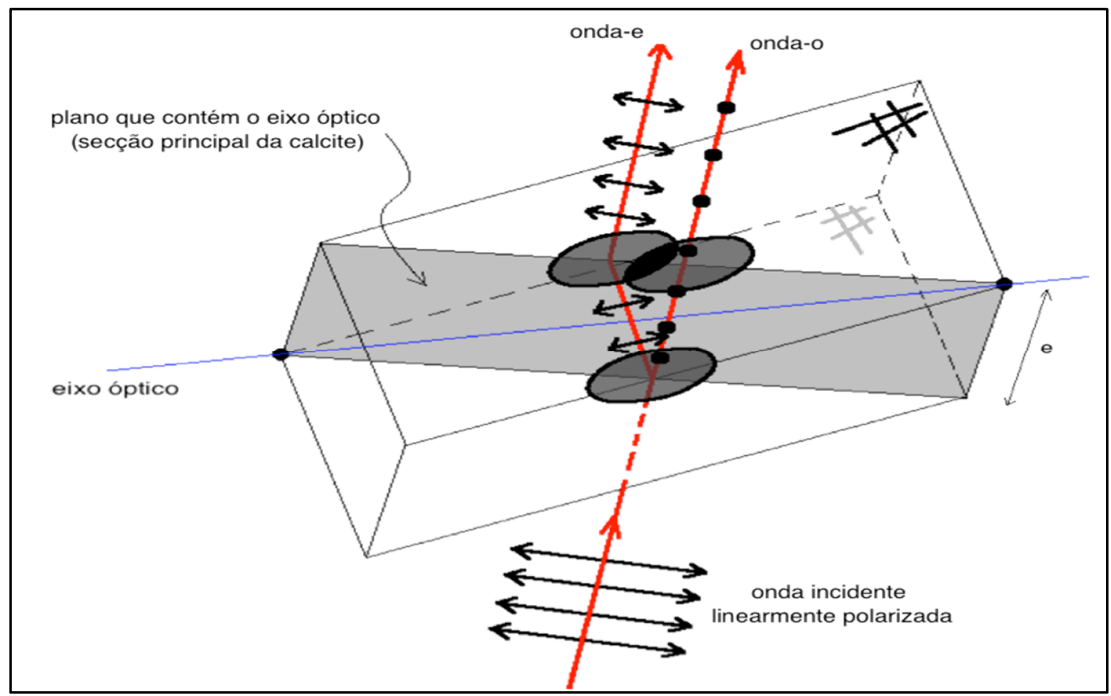

Fonte: Sidney Leal da Silva

Então, a diferença de índice de refração produzida pelo material faz com que as ondas tenham uma diferença de fase que resulta em um padrão de interferência para o observador.

Alguns materiais não cristalinos transparentes apresentam refração única quando estão livres de esforços externos, por isso são denominados isotrópicos. $\mathrm{Na}$ presença de esforços externos, no entanto, eles passam a ter dupla refração tornando-se anisotrópicos de acordo com DA SILVA (2017).

Esse fenômeno, denominado birrefringência temporária, está associado à elasticidade mecânica do material e, portanto, à capacidade de alteração de seu arranjo molecular, fato não possível em materiais cristalinos devido à sua estrutura mais rígida.

A observação do fenômeno da birrefringência temporária em meios transparentes se dá pelas técnicas de fotoelasticidade que utilizam polariscópios fundamentados nas propriedades da luz polarizada em sua operação. Birrefringência e Fotoelasticidade são descritas com detalhamento nos trabalhos de SALEH (2007), FERREIRA (2003), HECTH (2002), DA SILVA (2017) e FREIRE (1987).

\section{LEI ÓPTICA DA TENSÃO}

Para um material fotoelástico que exibe o efeito da birrefringência temporária sob a ação de esforço externo, a lei óptica da tensão relaciona as alterações no índice de refração com o seu estado plano de tensões.

Essas alterações são linearmente proporcionais ao esforço mecânico externo sobre o material e, consequentemente, esses índices de refração estão associados às tensões e deformações em seu interior. 
A partir de um tensor que relaciona os índices de refração, $\left(n_{0}-n_{e}\right)$, com as diferenças de tensões no estado plano, $\left(\sigma_{0}-\sigma_{e}\right)$, tem-se a lei óptica da tensão dada por:

$$
\mathrm{n}_{\mathrm{o}}-\mathrm{n}_{\mathrm{e}}=\mathrm{C} \cdot\left(\sigma_{\mathrm{o}}-\sigma_{\mathrm{e}}\right)
$$

com $C=C(\lambda)$ representando a dispersão de birrefringência ou dispersão fotoelástica que é uma constante do material para um determinado comprimento de onda $(\lambda)$. Em termos práticos, para um material de espessura (e), a lei da expressão (2) pode ser apresentada como

$$
\left(\sigma_{\mathrm{o}}-\sigma_{\mathrm{e}}\right)=\frac{\mathrm{f}_{\sigma} \cdot \mathrm{N}}{\mathrm{e}}
$$

em que $f_{\sigma}$ representa 0 valor de franjas do material e $\mathrm{N}$ a retardação óptica relativa, tais que:

$$
\begin{gathered}
\mathrm{f}_{\sigma} \equiv \frac{\lambda}{\mathrm{C}} \\
\mathrm{N} \equiv \frac{\Delta \phi}{2 \pi}
\end{gathered}
$$

já que a diferença de fases $(\Delta \phi)$ está diretamente associada à diferença de índices de refração $\left(\mathrm{n}_{\mathrm{o}}-\mathrm{n}_{\mathrm{e}}\right)$ das ondas.

A partir da expressão (3), a diferença entre as tensões do estado plano pode ser calculada se 0 valor de franja do material $\left(f_{\sigma}\right)$ for determinado através dos parâmetros do material e se as retardações relativas (N) forem encontradas por algum dos métodos estabelecidos em fotoelasticidade.

Um desses métodos, denominado fotoelasticidade RGB, descrito com detalhes no trabalho de RAMESH (2000), possibilita encontrar os valores de $\mathrm{N} \mathrm{a}$ partir de uma tabela fotoelástica de referência.

0 valor de franjas $\left(\mathrm{f}_{\sigma}\right)$ é uma propriedade do material e indica sua maior ou menor rigidez. Associando esse valor à teoria de Elementos Finitos, detalhada no trabalho de SORIANO (2003), tem-se:

$$
\mathrm{f}_{\sigma}=\frac{\mathrm{e} \cdot \mathrm{E}}{1+v}
$$

sendo (E) o módulo de elasticidade e ( $v$ ) o coeficiente de Poisson do material. Essas ideias são apresentadas com maior profundidade nos trabalhos de DA SILVA (2017), SALEH (2007), FERREIRA (2003), HECTH (2002), SOARES (1997) e FREIRE (1987).

\section{MATERIAIS, TÉCNICAS E MÉTODOS}

\section{AMOSTRAS}

Foram confeccionados dois lotes de amostras da mesma substância: (i) amostras para determinações dos parâmetros mecânicos (P1 e P2) e (ii) amostras para determinações das distribuições de tensões birrefringentes (D1 e D2).

Página | 37 
A amostra D2 foi confeccionada com um parafuso metálico fixo em sua base superior. A Figura 2 apresenta as fotografias das amostras fotoelásticas com dimensões distintas.

Figura 2 - amostras fotoelásticas

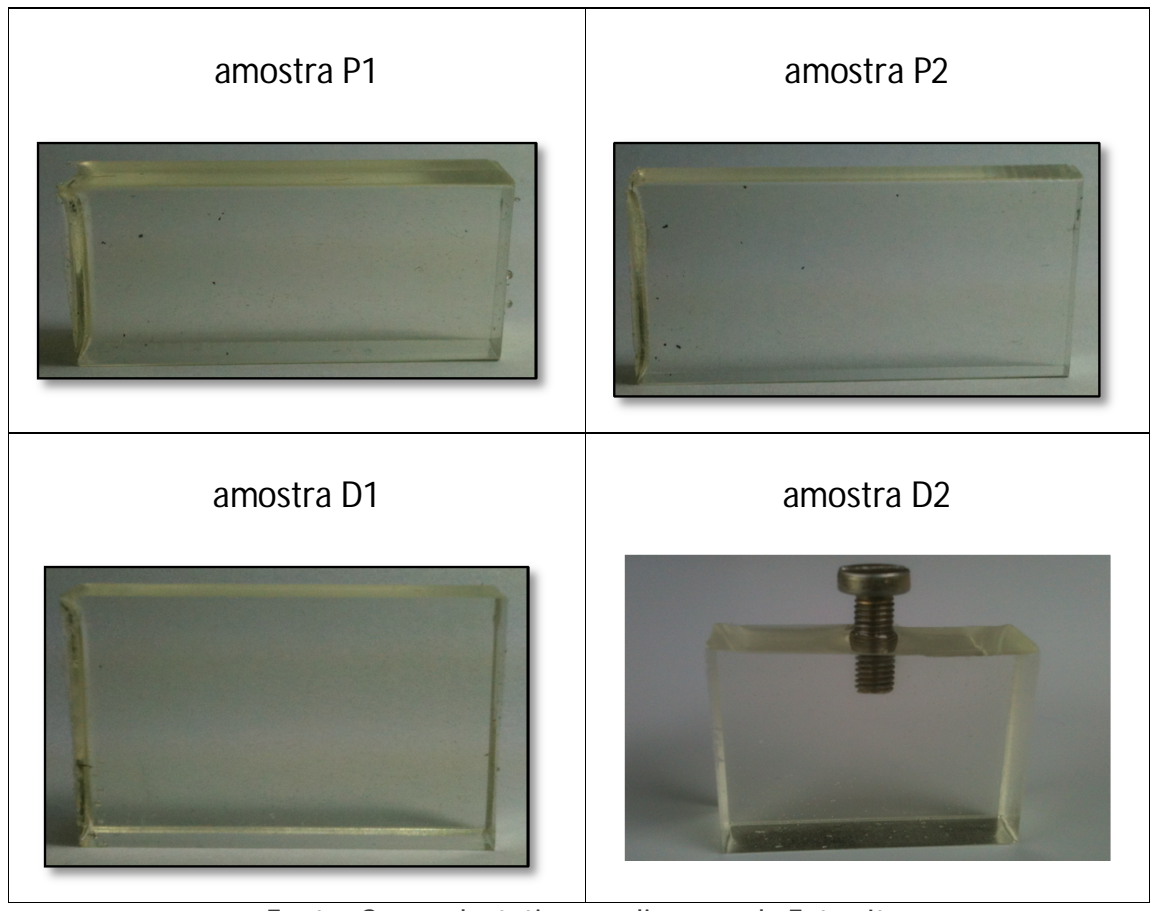

Fonte: Grupo de óptica e aplicações da Fatec Itaquera

\section{ENSAIO MECÂNICO}

Para os registros das imagens no ensaio mecânico, nas amostras P1 e P2, foi utilizada uma configuração experimental segundo o esquema apresentado na Figura 3: amostra fotoelástica submetida a esforços sucessivos. Uma lanterna possibilitou um melhor contraste da imagem registrada em uma câmera fotográfica.

Figura 3 - Esquema da configuração experimental do ensaio mecânico

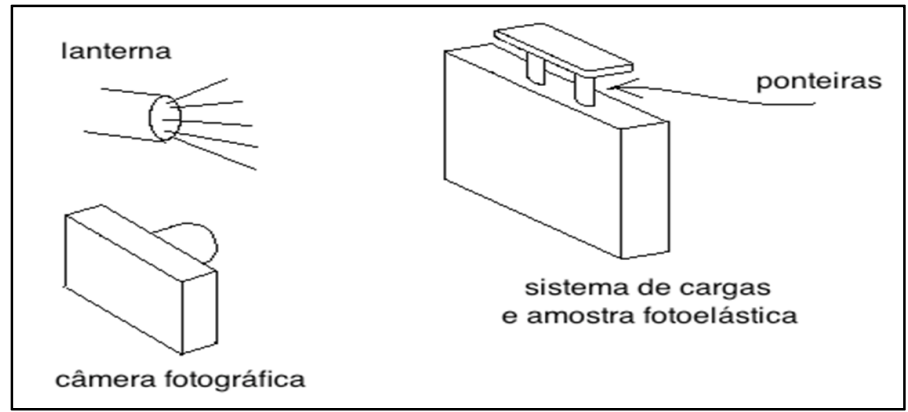

Fonte: Sidney Leal da Silva 
Em cada amostra fotoelástica foi aplicada uma sequência de $\mathbf{n}$ esforços externos verticais crescentes para levantamento dos dados de compressão e decrescentes para levantamento dos dados de descompressão. Foi também armazenada uma imagem de referência, sem esforço sobre a amostra.

\section{POLARISCÓPIO, REGISTROS DAS IMAGENS E CÁLCULOS DAS DEFORMAÇÕES}

A Figura 4 apresenta o esquema da configuração: fonte de luz branca com intensidade reduzida por um filtro de densidade neutra encontra um polarizador, antes de interagir com a amostra.

Em seguida, passa por outro polarizador, denominado analisador. Entre os polarizadores um dispositivo de carga permitiu aplicar esforço externo na amostra, alterando o estado de polarização da luz. Um filtro de cor (verde) selecionou somente as franjas com essa frequência para registro na câmera fotográfica.

Figura 4 - Esquema do polariscópio linear por transmissão

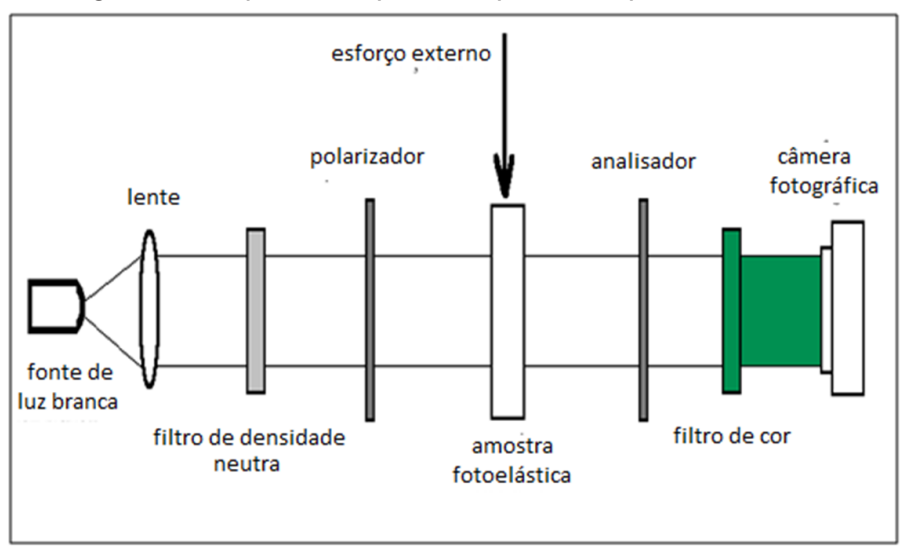

Fonte: Grupo de óptica e aplicações da Fatec Itaquera.

Os registros realizados com as amostras P1 e P2 foram utilizados para determinações das tabelas fotoelásticas de referências e com as amostras D1 e D2 para determinações das distribuições de tensões fotoelásticas.

Com as amostras P1 e P2 foram realizados os esforços externos. Foi utilizado um dispositivo de carga com uma base superior que recebeu, manualmente e progressivamente, as cargas responsáveis pelos esforços externos, durante 0 processo de compressão. Durante o processo de descompressão as cargas foram retiradas uma a uma para formar o conjunto de esforços externos regressivos.

Cada esforço gerou imagens em 8 bits armazenadas em arquivos. Também foram capturadas as imagens das amostras sem esforços externos, que serviram de referências para as medidas de deformações.

Computacionalmente foram lidas as alturas e larguras, em pixels, das amostras de todas as imagens para os $n$ esforços realizados. 
As deformações, tanto verticais $\left(\varepsilon_{\mathrm{V}}\right)$ quanto horizontais $\left(\varepsilon_{\mathrm{H}}\right)$ foram calculadas pelas diferenças relativas entre as alturas/larguras de duas imagens consecutivas, utilizando a altura/largura de referência.

Utilizando os valores das relações entre as tensões externas verticais $\sigma_{\mathrm{V}}$ versus $\varepsilon_{\mathrm{V}}$ e $\varepsilon_{\mathrm{H}}$ versus $\varepsilon_{\mathrm{V}}$, foram efetuados os ajustes a partir do polinômio de terceiro grau para verificar se a melhor função era linear, como prevê a lei de Hooke.

Após a verificação, os valores dos parâmetros que acompanharam as variáveis independentes de grau 1 forneceram os módulos de elasticidades para as relações $\sigma_{\mathrm{V}}$ versus $\varepsilon_{\mathrm{V}}$ e os coeficientes de Poisson para as relações $\varepsilon_{\mathrm{H}}$ versus $\varepsilon_{\mathrm{V}}$, nos dois processos: compressão e descompressão. A média entre os resultados encontrados nos dois processos produziu o resultado final desses parâmetros mecânicos para todas as amostras (1 e 2) de ambos os lotes.

\section{RESULTADOS E DISCUSSÕES}

\section{PARÂMETROS GEOMÉTRICOS DAS AMOSTRAS E ESFORÇOS EXTERNOS}

O Quadro 1 apresenta os valores dos parâmetros geométricos das amostras fotoelásticas P1, P2, D1 e D2, utilizadas para as determinações dos parâmetros mecânicos e das diferenças de tensões, respectivamente.

Quadro 1 - Parâmetros geométricos das amostras fotoelásticas P1, P2, D1 e D2

\begin{tabular}{|c|c|c|c|c|}
\hline & \multicolumn{4}{|c|}{$(\mathrm{cm})$} \\
\hline parâmetros & amostra P1 & amostra D1 & amostra P2 & amostra D2 \\
\hline comprimento & $3,959 \pm$ & $4,205 \pm$ & $5,580 \pm$ & $3,018 \pm$ \\
horizontal & 0,001 & 0,001 & 0,001 & 0,001 \\
\hline comprimento & $2,030 \pm$ & $3,026 \pm$ & $2,061 \pm$ & $2,609 \pm$ \\
vertical & 0,001 & 0,001 & 0,001 & 0,001 \\
\hline espessura & $1,025 \pm$ & $1,031 \pm$ & $1,039 \pm$ & $1,049 \pm$ \\
& 0,001 & 0,001 & 0,001 & 0,001 \\
\hline
\end{tabular}

Fonte: Grupo de Óptica e Aplicações da Fatec Itaquera

Para as determinações dos módulos de elasticidades e dos coeficientes de Poisson, as amostras P1 e P2 foram submetidas a uma sequência de esforços totais estáticos e homogêneos, segundo a configuração esquematizada na Figura 3, com as intensidades apresentadas no Quadro 2.

As massas utilizadas incluiram a do conjunto base de cargas, haste principal móvel e da base de contato com a amostra, de valor $m=(1,50 \pm 0,01) \cdot 10^{-1} \mathrm{~kg}$.

Os valores das forças foram calculados pelos produtos entre as massas e a aceleração da gravidade local da cidade de São Paulo, latitude média $23^{0} \mathrm{e}$ altitude média aproximadamente $760 \mathrm{~m}$ do nível do mar, $\mathrm{g}=(9,79 \pm 0,01) \mathrm{m} / \mathrm{s}^{2}$, calculado no trabalho de LOPES (2008). 
Quadro 2 - Esforços externos aplicados nas amostras P1 e P2

\begin{tabular}{|c|}
\hline $\begin{array}{c}\text { Tensão externa vertical } \\
(\mathrm{M} \mathrm{Pa})\end{array}$ \\
\hline$(3,77 \pm 0,07) \cdot 10^{-1}$ \\
\hline$(5,02 \pm 0,09) \cdot 10^{-1}$ \\
\hline$(6,28 \pm 0,11) \cdot 10^{-1}$ \\
\hline$(7,53 \pm 0,14) \cdot 10^{-1}$ \\
\hline$(8,79 \pm 0,16) \cdot 10^{-1}$ \\
\hline $1,00 \pm 0,02$ \\
\hline $1,13 \pm 0,02$ \\
\hline $1,26 \pm 0,02$ \\
\hline $1,38 \pm 0,03$ \\
\hline $1,51 \pm 0,03$ \\
\hline
\end{tabular}

Fonte: Grupo de Óptica e Aplicações da Fatec Itaquera

As amostras D1 e D2 foram submetidas ao esforço em massa de $(6,00 \pm$ $0,01) \cdot 10^{-1} \mathrm{~kg}$, que equivale à força de $(5,87 \pm 0,01) \mathrm{N}$, com as tensões apresentadas no Quadro 3.

Quadro 3 - Esforços externos aplicados nas amostras fotoelásticas D1 e D2

\begin{tabular}{|c|c|}
\hline Tensão externa & vertical (M Pa) \\
\hline amostra D1 & amostra D2 \\
\hline$(5,45 \pm 0,04) \cdot 10^{-1}$ & $1,03 \pm 0,02$ \\
\hline
\end{tabular}

Fonte: Grupo de Óptica e Aplicações da Fatec Itaquera

\section{MÓDULOS DE ELASTICIDADES E COEFICIENTES DE POISSON}

As Figuras 5 e 6 apresentam os gráficos experimentais das tensões externas versus deformações lineares longitudinais, a partir dos dados obtidos nos ensaios de compressão e descompressão sobre a amostra fotoelástica P1.

Figura 5 - Gráfico experimental das tensões externas (compressõ es) versus deformações longitudinais

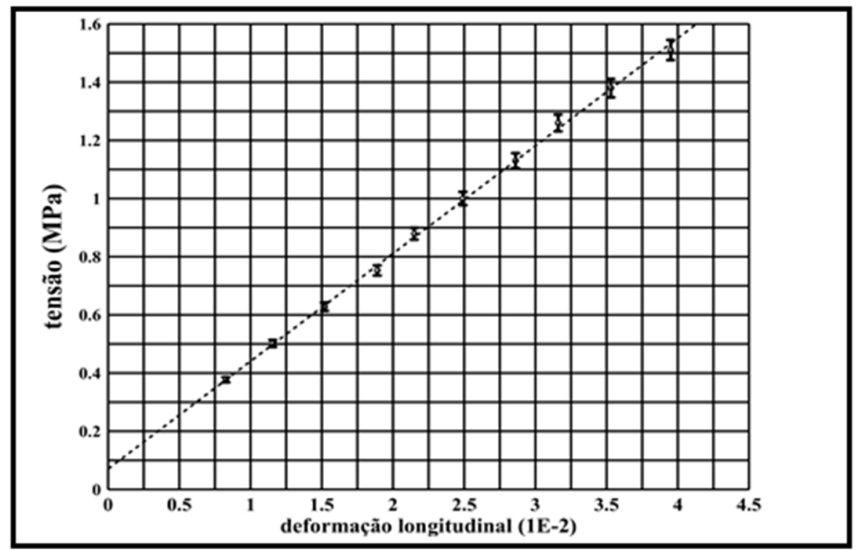

Fonte: Grupo de Optica e Aplicações da Fatec Itaquera

Página | 41 
Figura 6 - Gráfico experimental das tensões externas (descompressões) versus deformações longitudinais

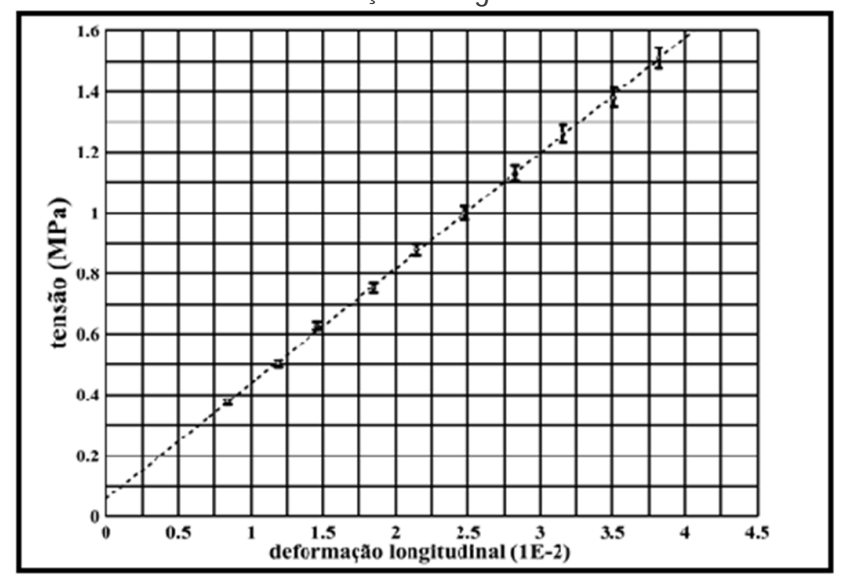

Fonte: Grupo de Óptica e Aplicações da Fatec Itaquera

Os valores dos módulos de elasticidade (E) foram obtidos a partir dessas funções e os valores finais, $\mathbb{E}$, resultaram da média entre os valores de compressão e descompressão. 0 Quadro 4 apresenta os resultados obtidos para as amostras P1 e P2.

Quadro 4 - Módulos de elasticidade para as amostras P1 e P2

\begin{tabular}{|c|c|}
\hline amostra & $\varangle>10^{-2} \mathbf{~ G P a}$ \\
\hline P1 & $3,74 \pm 0,37$ \\
\hline P2 & $3,86 \pm 0,27$ \\
\hline
\end{tabular}

Fonte: Grupo de Óptica e Aplicações da Fatec Itaquera

As Figuras $\mathbf{7}$ e $\mathbf{8}$ apresentam os gráficos experimentais das deformações lineares perpendiculares ao esforço versus deformações lineares longitudinais.

Figura 7 - Gráfico experimental das deformações perpendiculares (compressão) versus longitudinais

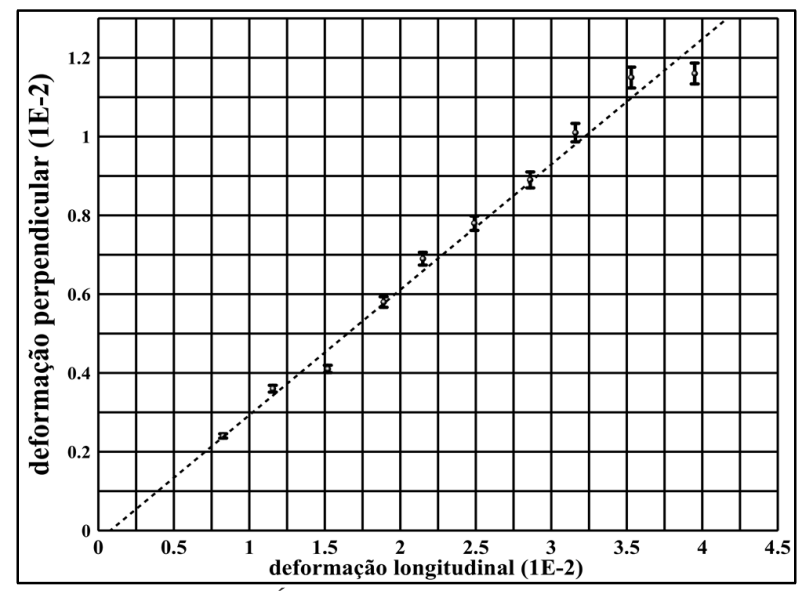

Fonte: Grupo de Óptica e Aplicações da Fatec Itaquera

Página | 42 
Figura 8 - Gráfico experimental das deformações perpendiculares (descompressão) versus longitudinais

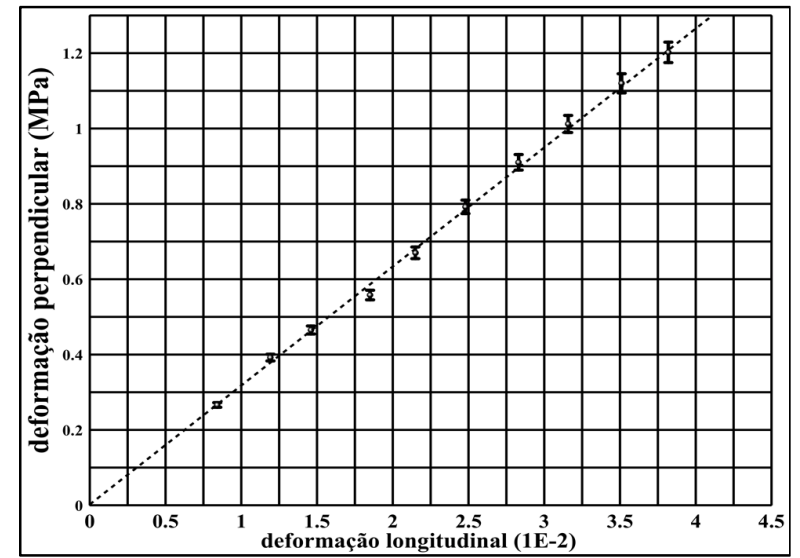

Fonte: Grupo de Óptica e Aplicações da Fatec Itaquera

Os gráficos foram traçados a partir dos dados obtidos nos ensaios de compressão e descompressão sobre a amostra fotoelástica P1. As linhas tracejadas são guias para obervação das distribuições dos valores experimentais. Utilizando o método dos mínimos quadrados foram obtidos os ajustes das funções para a compressão e a descompressão.

Os valores dos coeficientes de Poisson, $v$, foram obtidos a partir dessas funções e os valores finais, $\langle v\rangle$, resultaram da média entre os valores de compressão e descompressão. 0 Quadro 5 apresenta os resultados obtidos para as amostras P1 e P2.

Quadro 5 - Coeficientes de Poisson (adimensional) para as amostras P1 e P2

\begin{tabular}{|c|c|}
\hline Amostra & $<v>1 \mathbf{1 0}^{-1}$ \\
\hline P1 & $3,222 \pm 0,012$ \\
\hline P2 & $3,511 \pm 0,011$ \\
\hline
\end{tabular}

Fonte: Grupo de Óptica e Aplicações da Fatec Itaquera

Os resultados dos Quadros 4 e $\mathbf{5}$ foram utilizados para determinações das distribuições teóricas de tensões, nos cálculos dos valores de franjas em fotoelasticidade.

\section{FOTOELASTICIDADE}

\section{VALORES DE FRANJAS (f $\left.\mathbf{f}_{\sigma}\right)$ E DISPERSÕES FOTOELÁSTICAS (C)}

Nas amostras D1 e D2 foram realizados os esforços externos apresentados no Quadro 3, que geraram imagens em 8 bits, armazenadas em arquivos. A amostra D1 é uma amostra pura, livre de peça metálica.

Página | 43 
Na amostra D2, durante a cura, foi inserido um parafuso metálico. Para as determinações dos valores de franjas das amostras D1 e D2 foram utilizados os resultados médios dos módulos de elasticidade do QUADRO 4, dos coeficientes

de Poisson do Quadro 5, as espessuras das amostras do Quadro 1 e a expressão (5). 0 Quadro 6 agrupa todos os resultados.

Quadro 6 - Valores de franjas das amostras D1 e D2

\begin{tabular}{|c|c|}
\hline amostra & $\mathrm{f}_{\sigma}\left(\mathbf{1 0 ^ { \mathbf { 5 } }} \mathbf{N} / \mathbf{m}\right)$ \\
\hline D1 & $2,92 \pm 0,29$ \\
\hline D2 & $3,00 \pm 0,21$ \\
\hline
\end{tabular}

Fonte: Grupo de Óptica e Aplicações da Fatec Itaquera

A fim de se determinarem as dispersões fotoelásticas (C) das amostras D1 e D2, utilizaram-se os valores de franjas do Quadro 6, o comprimento de onda médio da luz verde $\lambda=532,5 \mathrm{~nm}$ e a definição de valor de franja fotoelástica, expressão (4a). Os resultados estão apresentados no Quadro 7, abaixo.

Quadro 7 - Dispersões fotoelásticas das amostras fotoelásticas

\begin{tabular}{|c|c|}
\hline amostra & $\mathrm{C}\left(\mathbf{1 0} \mathbf{0}^{-12} \mathbf{~ m}^{2} / \mathbf{N}\right)$ \\
\hline D1 & $1,82 \pm 0,18$ \\
\hline D2 & $1,78 \pm 0,12$ \\
\hline
\end{tabular}

Fonte: Grupo de Óptica e Aplicações da Fatec Itaquera

\section{TABELAS FOTOELÁSTICAS DE REFERÊNCIAS}

A Figura 9 mostra as franjas selecionadas das imagens em tons de cinza e coloridas das amostras fotoelásticas P1 e P2, submetidas aos esforços em massa de $(1,20 \pm 0,01) \mathrm{kg}$ em um polariscópio linear.

Neste experimento, o filtro verde foi o que proporcionou um melhor contraste das imagens das franjas ordenadas entre $[0,3]$.

Figura 9 - Trechos selecionados das imagens obtidas no polariscópio

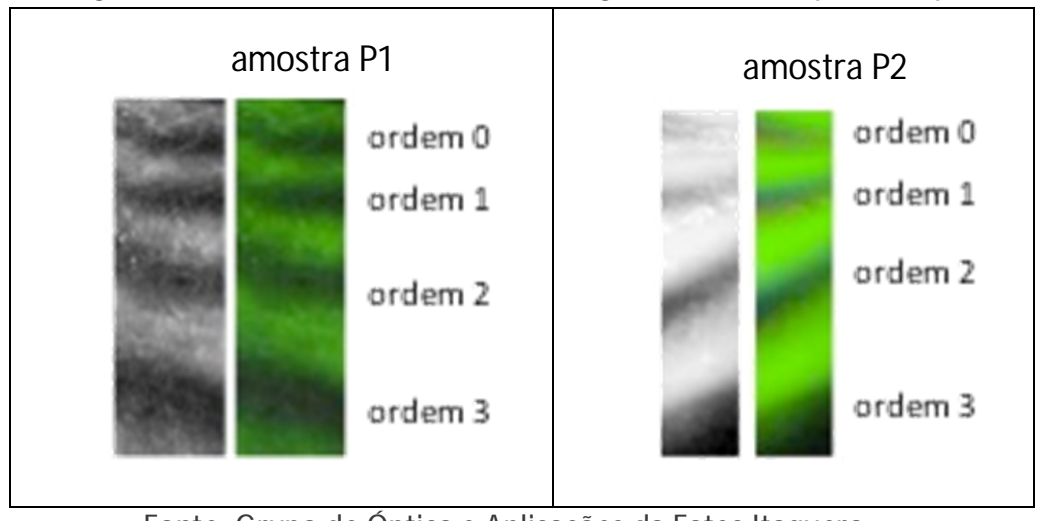

Fonte: Grupo de Óptica e Aplicações da Fatec Itaquera 
Após a análise e normalizações, os resultados foram armazenados em arquivos e serviram como tabelas de referências para determinações das retardações relativas, $\mathrm{N}_{\mathrm{j}}$, em cada pixel j da região selecionada das imagens de franjas obtidas com as amostras D1 e D2.

\section{DISTRIBUIÇÕES FOTOELÁSTICAS DAS DIFERENÇAS DE TENSÕES}

Os trechos selecionados para determinações das distribuições de diferenças de tensões foram retângulos entre as franjas escuras das imagens das amostras D1 e D2.

As imagens foram obtidas em um polariscópio linear. As duas amostras foram submetidas ao esforço externo, em massa, de $(6,00 \pm 0,01) \cdot 10^{-1} \mathrm{~kg}$.

A Figura 10 apresenta as imagens selecionadas das amostras D1 e D2. As linhas brancas identificam aproximadamente os trechos estudados.

Figura 10 - Trechos (retêngulos) para determinações das diferenças de tensões

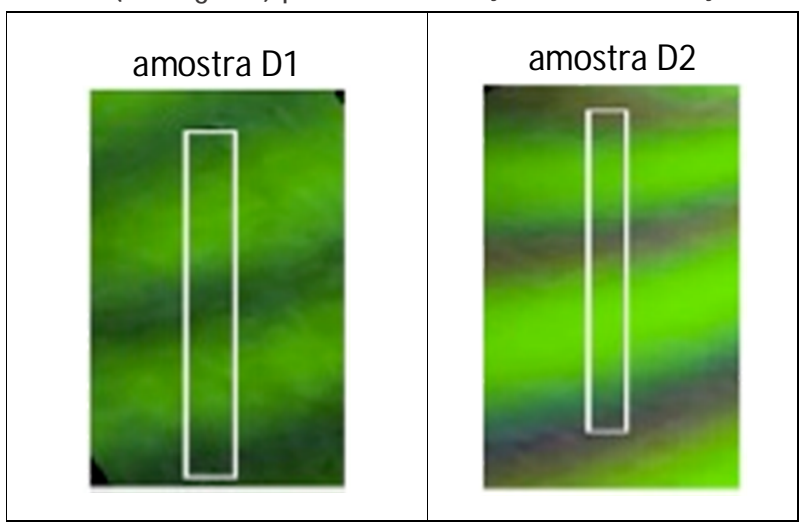

Fonte: Grupo de Óptica e Aplicações da Fatec Itaquera

Foram determinadas, a partir das imagens fotoelásticas, as retardações relativas médias, $\mathrm{N}_{\mathrm{j}}$. Com os valores dos Quadros 4 e 5, encontrou-se a rigidez $(\mathrm{f} \sigma / \mathrm{e})$, de cada amostra e com a expressão (4) foram calculadas as distribuições das diferenças de tensões para as amostras D1 e D2.

Os resultados finais foram organizados em gráficos das distribuições das diferenças de tensões versus pixel vertical nas amostras fotoelásticas D1 (esquerda) e D2 (direita) nos trechos verticais médios entre as duas primeiras franjas escuras, como mostram as Figuras 11 e 12.

As amostras D2 já apresentam tensões residuais devido à peça de metal inserida na resina. Estas tensões residuais são acrescidas às tensões provocadas pelo esforço externo, que é o mesmo aplicado na amostra D1. Isso explica o fato das distribuições de tensões da amostra D1 apresentarem picos menores e mais espalhados em relação às distribuições de tensões das amostras D2. 
Figura 11 - Distribuições de diferenças de tensões versus pixel vertical (franja 1)
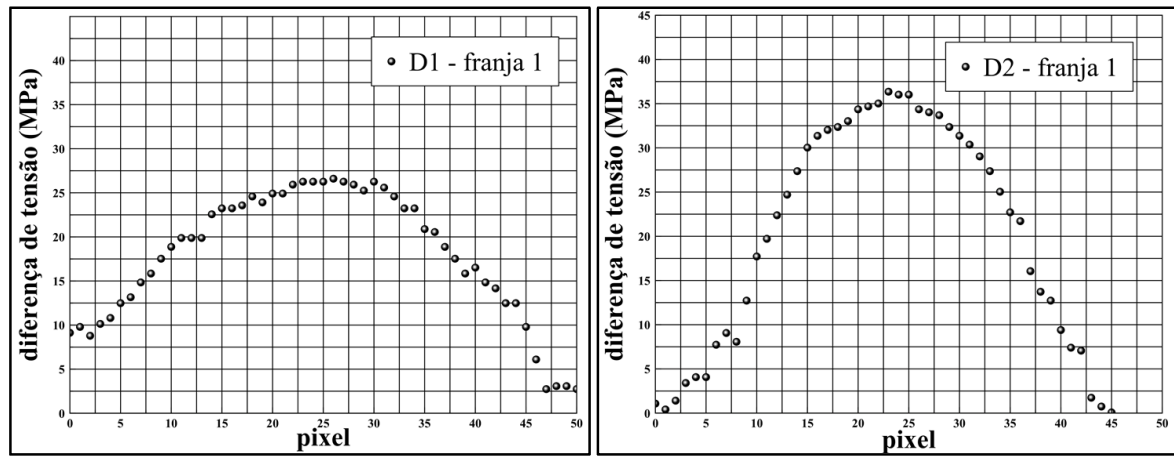

Fonte: Grupo de Óptica e Aplicações da Fatec Itaquera

Figura 12 - Distribuiç̧ões de diferenças de tensões versus pixel vertical (franja 2)
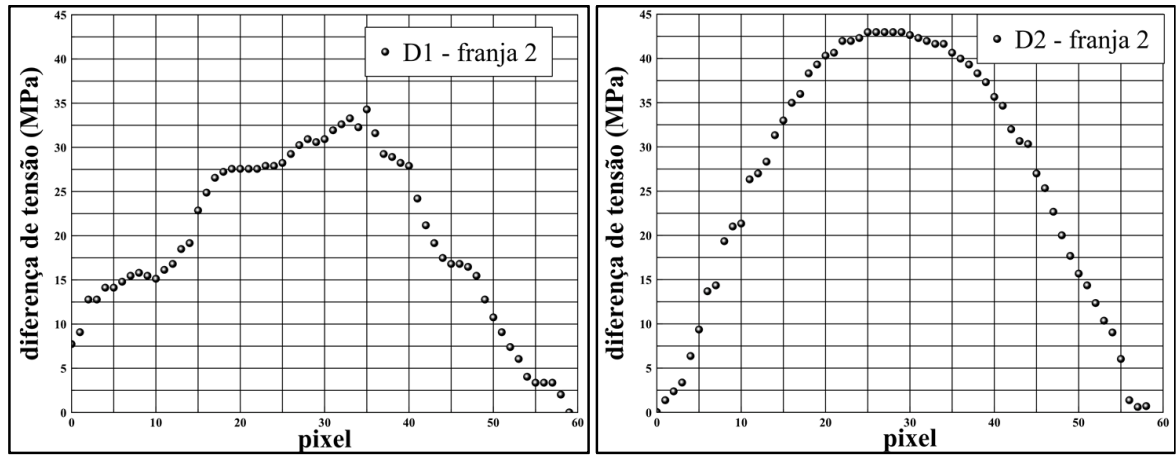

Fonte: Grupo de Óptica e Aplicações da Fatec Itaquera

Em cada amostra, separadamente, as segundas franjas apresentam picos maiores e mais espalhados em relação às primeiras franjas. Isso mostra que a distribuição tende a se intensificar para ordens maiores de franjas, DA SILVA (2017). 


\section{CONSIDERAÇÕES FINAIS}

Determinou-se, por meio do estudo apresentado, a distribuição quantitativa de tensões em amostras fotoelásticas e, por meio da comparação entre essas distribuições, verificou-se as alterações provocadas ao incorporar uma peça metálica à resina.

Uma peça inserida no material fotoelástico provoca tensões residuais, já que o estado de polarização também é alterado qualitativamente, como mostraram as alterações das distribuições de tensões das Figuras 11 e 12.

Só foi possível analisar as imagens a partir da utilização do filtro de cor verde, já que as imagens policromáticas continham muitas franjas isoclínicas (escuras), que causaram dificuldades na visualicação das franjas mais centrais.

Entende-se que resultados ainda mais significativos poderiam ser obtidos se fossem acrescidas ao sistema lâminas de quarto de onda na construção de um polariscópio elíptico para permitir somente a visualização de franjas isocromáticas. Por meio dessas lâminas seria possível também efetuar a análise das imagens policromáticas. Para que isso seja possível, o grupo de pesquisa deverá captalizar recursos para compra de equipamentos.

Observa-se como possibilidade de ampliar os conhecimentos sobre análise de tensões a realização de um estudo a fim de determinar as propriedades do material, módulo de elasticidade e coeficiente de Poisson, para utilização em aplicações que envolvem fraturas e resistência dos materiais. 


\title{
Quantitative Analysis of Stresses in Photoelastic Samples by Means of Photoelasticity
}

\begin{abstract}
Photoelasticity is an efficient conduit for the determination of tensions in photoelastic materials. These materials exhibit the property of birefringence when subjected to external stresses due to changes in the polarization states of light transmitted through its structure. From a linear polariscope of transmission it is possible to obtain colored images of fringes that are used to find the differences of tensions by means of analysis with the method of RGB photoelasticity. The objective of this work is to determine the distributions of these stresses differences in two consecutive fringes of monochromatic images produced with the help of a green filter in photoelastic samples: pure and with a metallic screw inserted in the structure. This leads to results that indicate the possibility of a quantitative analysis in the study of the properties of the materials by a Photoelasticity method slightly modified in comparison to the traditional method; therefore, a nondestructive optical method.
\end{abstract}

KEYWORDS: Photoelastic materials; Photoelasticity; Polarization; Birefringence. 


\section{El análisis cuantitativo de estrés fotoelástico por medio de fotoelasticidad}

\section{RESUMEN}

Fotoelasticidad es una herramienta eficaz para determinar las tensiones dentro de los materiales fotoelásticos. Estos materiales muestran la propiedad de birrefringencia temporal, cuando se someten a estreses externos, debido a cambios en los estados de polarización de la luz transmitida a través de la estructura. Desde un polariscopio de transmisión lineal, es posible obtener imágenes de flecos en colores que se utilizan para encontrar las diferencias de estreses por el análisis con el método RGB fotoelasticidad. El objetivo de este estudio es determinar las distribuciones de las diferencias de estrese en dos flecos sucesivos de imágenes monocromáticas que se producen con la ayuda de un filtro verde en muestras fotoelásticas: pura y con un tornillo de metal que se inserta en la estructura de las muestras. Ello conduce a resultados que indican la posibilidad de un análisis cuantitativo en el estudio de las propiedades del material por el método óptico no destructivo.

PALABRAS CLAVE: Materiales fotoelásticos; Fotoelasticidad; Polarización; Birrefringencia. 


\section{AGRADECIMENTOS}

Os autores agradecem ao Grupo de óptica e aplicações, GOA, à Câmara de Ensino, Pesquisa e Extensão, CEPE, e à Direção da Fatec Itaquera pelo incentivo e apoio

\section{REFERÊNCIAS}

CARLIN, N. et al. Birrefringência em placas de onda e atividade óptica de uma solução de açúcar. Revista Brasileira de Ensino de Física, v. 27, n. 3, p. 349-355, 2005.

DA SILVA, S. L. et al. Análise qualitativa de tensões em estruturas sólidas acopladas a amostras de resina fotoelástica. Revista Brasileira de Física Tecnológica Aplicada, v. 2, n. 1, p. 1-13, 2015.

DA SILVA, S. L. Estudo de tensões em amostras fotoelásticas com Holografia Digital - Análise quantitativa. 1ํ Edição: Novas Edições Acadêmicas, 2017, Brasil. $153 p$.

FERREIRA, M . Ótica e Fotônica, Editora Lidel, 2003 Lisboa - Portugal.

FREIRE, J. L. F. Introdução à Fotoelasticidade. Rio de Janeiro: PUC/RJ, Departamento de Engenharia M ecânica, 1987.

HECTH, E. Óptica, 2o edição, Fundação Calouste Gulbenkian, 2002, Lisboa, Portugal.

KALE, S.; RAMESH, K. Advancing front scanning approach for three-fringe photoelasticity. Optics and Lasers in Engineering, v. 51, n. 5, p. 592-599, 2013.

KUSKE, A.; ROBERTSON, G. Photoelastic Stress Analysis. New York: John Wiley and Sons, 1974.

LOPES, W. Variação da Aceleração da Gravidade com a Latitude e Altitude. Caderno de Ensino de Física, v. 25, n.3, p. 561-568, 2008. ISSN-e 2175-7941.

MAIA, L. G. M. et al. Qualitative photoelastic study of the force system produced by retraction T-springs with diferente preactivations. Dental Press Journal of Orthondontics. 2010, v. 15, n. 4, p. 103-116.

NETO, B. G. et al. Análise de controle de escoamento em areronaves usando fotoelasticidade para verificação experimental de protótipos feitos de resinas transparentes. Revista Interdisciplinar de Pesquisa em Engenharia - RIPE. 2016, v. 2, n. 23, p. 103-116.

RAMESH, K. Digital Photoelasticity. Measurement Science and Technology, v. 11, n. 12.

SALEH, B. E. A.; TEICH, M. C. Fundamentals of Photonics. Second Edition. Canada: John Wiley \& Sons, Inc., 2007, 1177 p. 
SOARES, W. A. Determinação de parâmetros da mecânica de fratura a partir de imagens fotoelásticas, usando processamento digital. 1997. Tese de Doutorado. Instituto de Pesquisas Energéticas e Nucleares, São Paulo.

SORIANO, H. L. Método de Elementos Finitos em Análise de Estruturas. Primeira Edição. São Paulo: Editora Edusp, 2003. 580 p.

SWAIN, D. et al. Novel calibration and color adaptation schemes in three-fringe RGB photoelasticity. Optics and Lasers in Engineering, v. 66, p. 320-329, 2015.

VUOLO, J. H. Fundamentos da Teoria de Erros. São Paulo: Editora Edgard Blucher Ltda, 1992. 225 p.

ZHANG, Y. et al. Branch cutting algorithm for unwrapping photoelastic phase map with isotropic point. Optics and Lasers in Engineering, v. 50, n. 5, p. 619-631.

Recebido: 04 de fevereiro de 2017

Aprovado: 25 de abril de 2017.

Dol:

Como citar: SILVA, L.S. et al, Análise quantitativa de tensões em amostras fotoelásticas por meio de fotoelasticidade, Revista Brasileira de Física Tecnológica Aplicada, Ponta Grossa, v. 4, n.1, p. 34-51,

mai./ jun. 2017

Contato:Sidney Leal Silva: fatec.professor.sidney@gmail.com

Direito autoral: Este artigo está licenciado sob os termos da Licença Creative Commons-Atribuição 4.0 Internacional.

(c) (i) 